\title{
NONNEGATIVE SOLUTIONS FOR A HETEROGENEOUS DEGENERATE COMPETITION MODEL
}

\author{
ANTONIO SUÁREZ 1
}

(Received 5 February, 2003; revised 10 October, 2003)

\begin{abstract}
This paper deals with the existence, uniqueness and qualitative properties of nonnegative and nontrivial solutions of a spatially heterogeneous Lotka-Volterra competition model with nonlinear diffusion. We give conditions in terms of the coefficients involved in the setting of the problem which assure the existence of nonnegative solutions as well as the uniqueness of a positive solution. In order to obtain these results we employ monotonicity methods, singular spectral theory and a fixed point index.
\end{abstract}

\section{Introduction}

In this work we are mainly concerned with the existence and uniqueness of nonnegative solutions for the problem

$$
\begin{cases}L_{1}\left(w^{m}\right)=w(\lambda-a(x) w-b(x) z) & \text { in } \Omega, \\ L_{2}\left(z^{n}\right)=z(\mu-d(x) z-c(x) w) & \text { in } \Omega, \\ w=z=0 & \text { on } \partial \Omega\end{cases}
$$

where $\Omega$ is a bounded domain of $\mathbb{R}^{N}$ with regular boundary $\partial \Omega, L_{k}, k=1,2$, are two second-order uniformly elliptic operators of the form

$$
L_{k}:=-\sum_{i, j=1}^{N} a_{i j}^{k}(x) D_{i} D_{j}+\sum_{i=1}^{N} b_{i}^{k}(x) D_{i} \quad k=1,2,
$$

with $a_{i j}^{k}, b_{i}^{k} \in C^{1}(\bar{\Omega}) ; m, n>1 ; \lambda, \mu \in \mathbb{R}$ and $a, b, c, d \in C^{l}(\bar{\Omega})$ nonnegative and nontrivial.

\footnotetext{
${ }^{1}$ Dpto. Ecuaciones Diferenciales y Análisis Numérico, Fac. Matemáticas, C/ Tarfia s/n, C.P. 41012 , Univ. Sevilla, Sevilla, Spain; e-mail: suarez@us.es.

(C) Australian Mathematical Society 2004, Serial-fee code 1446-1811/04
} 
Problem (1.1) provides us with the steady-state solutions to a related evolutionary problem, which models the behaviour of two competing species, with population densities $w(x)$ and $z(x)$, inhabiting $\Omega$. We refer to [13] for the meaning of each coefficient and details about the model.

When $m=n=1$ (linear diffusion), (1.1) has been extensively studied in recent years. In the case when $a, b, c$ and $d$ are strictly positive functions, see for example $[6-9,11,12,18-20,24,27,32]$ and the references therein. When $b$ and/or $c$ vanish in a domain of $\Omega$ (this means that, for instance, $z$ does not interact with $w$ in the set $\left.B_{0}:=\{x \in \Omega: b(x)=0\}\right)$, problem (1.1) was studied in [21,25] and [27]. And finally, recently the case when $a$ vanishes in a part of $\Omega$ but all other coefficients and functions are strictly positive over $\Omega$ has been analysed in [17] and [26], where essential qualitative changes occur. Observe that in this case positive constants are not supersolutions of (1.1) and, in fact, it is shown that the a priori bounds are lost for some values of $\lambda$ and $\mu$ and a new kind of positive solutions appear (which are infinite over a region of $\Omega$ and finite on the rest of $\Omega$ ) that govern the behaviour of a related evolutionary problem.

However, model (1.1) is less known when $m, n>1$, and it has only been analysed under more restrictive hypotheses, with constant coefficients (the homogeneous environmental case) in [13] and when $a$ and $d$ are strictly positive in [28] and [30], all of them with $L_{1}=L_{2}=-\Delta$. These new parameters $(m, n)$ were introduced in $[22,29]$ by describing the dynamics of a biological population whose mobility depends upon its density. In this context, it means that the diffusion, the rate of movement of the species from high density regions to low ones, is slower than in the linear case, giving more realistic results. Mathematically, this mainly has three consequences which distinguish this system from the one with $m=n=1$ : the strong maximum principle does not apply (and so, unlike the linear case, there can exist nonnegative and nontrivial solutions which are not positive in all $\Omega$ ), a priori bounds for all the solutions of (1.1) and for all the values of $\lambda$ and $\mu$, even when $a$ or $d$ vanishes, exist and that the linearised method cannot be applied directly.

In order to study (1.1) we make the appropriate change of variables $w^{m}=u$ and $z^{n}=v$, which transforms (1.1) into

$$
\begin{cases}L_{1} u=u^{1 / m}\left(\lambda-a(x) u^{1 / m}-b(x) v^{1 / n}\right) & \text { in } \Omega \\ L_{2} v=v^{1 / n}\left(\mu-d(x) v^{1 / n}-c(x) u^{1 / m}\right) & \text { in } \Omega, \\ u=v=0 & \text { on } \partial \Omega\end{cases}
$$

Since only nonnegative solutions have physical interest, there are four types of solutions: the trivial solution, the semitrivial solutions $(u, 0)$ and $(0, v)$, those with both components strictly positive, the coexistence states, and those where at least one component could vanish in a part of $\Omega$, the semicoexistence states. Observe that a 
semicoexistence state could also be a coexistence state (see Proposition 3.3). Sometimes, we are able to prove that a semicoexistence state vanishes in a region of $\Omega$ (see Theorem 3.4), and so it is not a coexistence state.

The paper is structured as follows. Observe that the semitrivial solutions satisfy the following equation, the reason for our study in Section 2,

$$
\begin{cases}L w=f(x) w^{1 / r}-g(x) w^{2 / r} & \text { in } \Omega, \\ w=0 & \text { on } \partial \Omega,\end{cases}
$$

where $L$ is an operator of the form (1.2), $f, g \in C^{1}(\bar{\Omega})$ with $g \geq 0, g \not \equiv 0, f$ can change sign and $r=m$ or $n$. Although the semitrivial solutions give $f \equiv \lambda$ (or $\mu$ ) and so constant, it will be very useful to study (1.4) when $f$ changes sign. This equation has been previously studied in $[3,13,14,23]$ and [31], assuming more restrictions on the data of (1.4). We collect the main results of these works, and as a consequence we obtain that the semitrivial solution $(u, 0)=$ (respectively $(0, v)$ ) exists and is unique if, and only if, $\lambda>0$ (respectively $\mu>0$ ).

We then study the existence of dead cores (see [16]) of the solutions of (1.4). Given a solution $w$ of (1.4); if the set $\Omega_{0}:=\{x \in \Omega: w(x)=0\}$ is nonempty, it is called a dead core of $w$. We demonstrate a result which assures the existence of a dead core for any nonnegative solution of (1.4) under suitable hypotheses (see Theorem 2.4). A direct consequence of our result is that any nonnegative solution of (1.4) has a dead core if the maximum of $f$ is small. To our knowledge, the above results pertaining to the existence of a dead core have been obtained when $L=-\Delta$, see [3], [13], [16] and [31], with their proofs being based on the radial properties of the Laplacian. In this way our result generalises previous work.

In Section 3 we carry out an analysis of the existence of semicoexistence, coexistence states and dead cores of the system (1.3). Using the results of Section 2 and monotonicity methods, we obtain results which can be summarised as follows. Suppose $\lambda \in \mathbb{R}$.

- Assume $\lambda \leq 0$ : if $\mu \in(-\infty, 0]$ only the trivial solution exists, if $\mu \in(0, \infty)$ only the trivial and the semitrivial solutions $(0, v)$ exist.

- Assume $\lambda>0$ : there exist positive values $\mu_{*}(\lambda), \mu^{*}(\lambda), \mu_{1}(\lambda), \mu_{2}(\lambda)$ with $\mu_{1}(\lambda)<\min \left\{\mu_{*}(\lambda), \mu^{*}(\lambda)\right\}$ and $\mu_{2}(\lambda)>\max \left\{\mu_{*}(\lambda), \mu^{*}(\lambda)\right\}$ such that

- if $\mu \in(-\infty, 0]$ only the trivial and semitrivial solution $(u, 0)$ exist;

- if $\mu \in\left(0, \mu_{1}(\lambda)\right)$ there exists at least a semicoexistence state $(u, v)$ and the component $v$ has a dead core;

- if $\mu \in\left(\mu_{1}(\lambda), \mu_{2}(\lambda)\right)$ there exists at least a semicoexistence state;

- if $\mu \in\left(\mu_{2}(\lambda), \infty\right)$ there exists at least a semicoexistence state $(u, v)$ and the component $u$ has a dead core;

- if, moreover $\mu_{*}(\lambda)<\mu^{*}(\lambda)$, then if $\mu \in\left(\mu_{*}(\lambda), \mu^{*}(\lambda)\right)$ there exists at least a 
coexistence state.

Analogous results can be obtained when we fix the parameter $\mu$. It's worth mentioning that the existence of $\mu_{1}(\lambda)>0$ was shown in [13] when all the coefficients were positive constants. To our knowledge, the existence of $\mu_{2}(\lambda)>0$ is new. In Remark 4 we give a biological interpretation of this result.

In Sections 4 and 5 we study the uniqueness of coexistence states of (1.3). For that we use the fixed point index. Observe that because $m, n>1$ the linearisation of (1.3) around the trivial or semitrivial solutions do not exist, so we cannot apply the results in [10] (see also [24] and [27]) to compute their indices. So we will build appropriate homotopies for that. To compute the index of a coexistence state we can use a linearisation. In this case the linearisation of (1.3) around a coexistence state leads us to a eigenvalue problem of the form

$$
\begin{cases}\mathscr{L} U+M U=\sigma U & \text { in } \Omega, \\ U=0 & \text { on } \partial \Omega,\end{cases}
$$

where $\mathscr{L}=\operatorname{diag}\left(L_{1}, L_{2}\right)$ and $M=\left(m_{i j}\right), 1 \leq i, j \leq 2$ with $m_{i j} \geq 0$ for $i \neq j$ and $m_{i j}$ blowing up near $\partial \Omega$ in a controlled way. Following [15] and [27] we define a specific order and establish the existence of the principal eigenvalue of (1.5) as well as a characterisation of its positivity by means of the existence of a supersolution. Now, we prove that, again with fixed $\lambda>0$, there exists a unique coexistence state when $\mu$ belongs to a subset of $\left(\mu_{*}(\lambda), \mu^{*}(\lambda)\right)$. Furthermore, if $m=n$ and $a, d$ are strictly positive functions we have uniqueness of the coexistence state if $b_{M}$ or $c_{M}$ is small. The results about the uniqueness of the coexistence state of (1.3) are also, we believe, new.

\section{Preliminaries. The degenerate logistic equation}

We consider the Banach space $X:=C_{0}^{1}(\bar{\Omega})$ ordered by its cone of nonnegative functions $P$, whose interior is

$$
\operatorname{int}(P):=\{u \in X: u(x)>0 \text { for all } x \in \Omega \text { and } \partial u / \partial n<0 \text { on } \partial \Omega\},
$$

where $n$ denotes the outward unit normal on $\partial \Omega$. We say that $u \in X$ is nonnegative, $u \geq 0$, if $u \in P$, and $u$ is positive, $u>0$, if $u \in \operatorname{int}(P)$.

Given $q \in L^{\infty}(\Omega)$ and $L$ an operator of the form (1.2), we denote by $\sigma_{1}(L+q)$ the principal eigenvalue of $L+q$ subject to homogeneous Dirichlet boundary conditions. Moreover, if we denote by $\varphi \in$ int $P$ the unique positive eigenfunction associated with $\sigma_{1}(L+q)$ normalised such that $\|\varphi\|_{\infty}=1$, then it is well known that

$$
\frac{\partial \varphi}{\partial \nu}<0 \text { on } \partial \Omega
$$


for $\nu$ any direction out of $\Omega$. Recall that as positive constants are supersolutions of $L$, then

$$
\sigma_{1}(L)>0 .
$$

Finally, for $f \in Y:=C^{0}(\bar{\Omega})$ we write

$$
f_{M}:=\max _{x \in \bar{\Omega}} f(x) \quad \text { and } \quad f_{L}:=\min _{x \in \bar{\Omega}} f(x) .
$$

2.1. Existence of solutions In this section we study the semitrivial solutions of (1.3). Observe that if the solutions of $(1.3)$ are of the form $(u, 0)$ and $(0, v)$, then they satisfy equations of the following type:

$$
\begin{cases}L w=f(x) w^{q}-g(x) w^{p} & \text { in } \Omega, \\ w=0 & \text { on } \partial \Omega,\end{cases}
$$

where $L$ is an operator of the form (1.2), $f, g \in C^{1}(\bar{\Omega})$ with $g \geq 0, g \not \equiv 0, f$ can change sign and $q$ and $p$ satisfy

$$
0<q<1, \quad p>q .
$$

Our first result gives us the existence of a nonnegative solution of (2.2) and lists some useful properties. For a proof of this result see [14] for instance.

THEOREM 2.1. Assume $(H)$. The following assertions are true.

(1) There exists a maximal nonnegative and nontrivial solution of (2.2) if, and only if, $f_{M}>0$. We denote it by $\theta_{[L, q, p, g, g]}$.

(2) The following estimates hold:

$$
\begin{aligned}
& \theta_{[L, q, p, f, g]}(x) \leq f_{M}^{1 /(1-q)} e_{M}^{q /(1-q)} e(x) \quad x \in \Omega, \\
& \left(\theta_{[L, q, p, f, g]}\right)_{M} \leq\left(f_{M} e_{M}\right)^{1 /(1-q)},
\end{aligned}
$$

where $e \in C^{2}(\bar{\Omega})$ is the unique solution of

$$
\begin{cases}L e=1 & \text { in } \Omega \\ e=0 & \text { on } \partial \Omega .\end{cases}
$$

(3) If $\underline{w} \in C^{1}(\bar{\Omega})$ is a nonnegative subsolution of (2.2), then $\underline{w} \leq \theta_{[L, q, p, f, g]}$.

(4) Let $f_{i} \in C^{\mathrm{l}}(\bar{\Omega}), i=1,2$, be such that $f_{1} \leq f_{2}$, then $\theta_{\left[L, q, p, f_{1}, g\right]} \leq \theta_{\left[L, q, p, f_{2}, g\right]}$.

(5) If $f_{L}>0$, then any nonnegative solution of (2.2) is positive. Moreover, in this case there exists a unique positive solution and it satisfies

$$
\varepsilon \varphi(x) \leq \theta_{[L, q, p, f, g]}(x), \quad x \in \Omega,
$$

where $\varepsilon$ is the unique positive root of $\sigma_{1}(L) \varepsilon^{1-q}+g_{M} \varepsilon^{p-q}=f_{L}$. 
REMARK 1. If we consider $f_{L}$ as a real parameter, then it is easy to prove that as $f_{L} \rightarrow \infty, \varepsilon\left(f_{L}\right)=O\left(f_{L}^{1 /(1-q)}\right)$ when $p \leq 1$ and $\varepsilon\left(f_{L}\right)=O\left(f_{L}^{1 /(p-q)}\right)$ when $p>1$.

2.2. Existence of dead cores In order to state and prove the main result, we need some preliminary results.

LEMMA 2.2. Let $R>0$ and $\gamma>0$. Consider the problem

$$
\begin{cases}L w=-R w^{q}-g(x) w^{p} & \text { in } \Omega \\ w=\gamma & \text { on } \partial \Omega\end{cases}
$$

Then there exists a unique nonnegative solution of (2.5).

PROOF. For the existence we use the sub-supersolution method. Indeed, it is easy to prove that $(\underline{w}, \bar{w})=(0, \gamma)$ is a sub-supersolution of $(2.5)$. For the uniqueness we can apply [1, Theorem 2$]$.

The following technical result is fundamental in our study. Moreover, it generalises [31, Lemma 7] and [3, Lemma 2.5], where a similar result was proved when $L=-\Delta$ and $g(x) \equiv 0$.

LEMMA 2.3. We fix $\gamma>0$ and $\beta>2 /(1-q)$. Let $\delta_{0}$ be such that for all $x, x_{0} \in \mathbb{R}^{N}$ such that $0 \leq\left|x-x_{0}\right| \leq \delta_{0}$,

$$
\begin{aligned}
& \left|x-x_{0}\right|^{\beta q}+\beta\left|x-x_{0}\right|^{\beta-1} L\left(\left|x-x_{0}\right|\right) \\
& \quad+\beta(1-\beta)\left|x-x_{0}\right|^{\beta-2} \sum_{i, j=1}^{N} a_{i j}(x) D_{i}\left(\left|x-x_{0}\right|\right) D_{j}\left(\left|x-x_{0}\right|\right) \geq 0 .
\end{aligned}
$$

Then, for all $0<\delta<\operatorname{dist}\left(x_{0}, \partial \Omega\right)$, the unique nonnegative solution, $w$, of (2.5) in $B\left(x_{0}, \delta\right)$ is such that $w\left(x_{0}\right)=0$ provided that

$$
R \geq\left(\frac{\gamma}{\min \left\{\delta, \delta_{0}\right\}^{\beta}}\right)^{1-q}
$$

REMARK 2. Observe that since $\beta>2 /(1-q)$, then $\beta q<\beta-2<\beta-1$, and so the existence of $\delta_{0}$ satisfying (2.6) is guaranteed. Moreover, since $\beta>2$, (2.6) can be considered in a classical sense.

Proof. Consider the function

$$
\Phi(x):= \begin{cases}\Phi_{1}(x):=R^{1 /(1-q)}\left|x-x_{0}\right|^{\beta} & \text { if } x \in \bar{B}\left(x_{0}, \delta_{0}\right), \\ \Phi_{2}(x):=R^{1 /(1-q)} \delta_{0}^{\beta} & \text { if } x \in B\left(x_{0}, \delta\right) \backslash B\left(x_{0}, \delta_{0}\right),\end{cases}
$$


with $\Phi \equiv \Phi_{1}$ if $\delta \leq \delta_{0}$. By the choice of $\beta$, we have that $\Phi_{1} \in H^{2}\left(B\left(x_{0}, \delta_{0}\right)\right)$. Moreover, $\partial \Phi_{1} / \partial n_{L} \geq 0$, on $\partial B\left(x_{0}, \delta_{0}\right)$, where $n_{L}$ stands for the conormal associated with $L$, that is, $\left(n_{L}\right)_{i}:=\sum_{j=1}^{N} a_{i j} n_{j}$. Indeed, for $x \in \partial B\left(x_{0}, \delta_{0}\right)$ we have

$$
\frac{\partial \Phi_{1}}{\partial n_{L}}(x)=R^{1 /(1-q)} \beta\left|x-x_{0}\right|^{\beta-3}\left(\sum_{i, j=1}^{N} a_{i j}(x)\left(x^{i}-x_{0}^{i}\right)\left(x^{j}-x_{0}^{j}\right)\right) \geq 0 .
$$

Moreover,

$$
\begin{aligned}
L\left(\Phi_{1}\right)+ & R \Phi_{1}^{q}+g(x) \Phi_{1}^{p} \\
= & R^{1 /(1-q)}\left(\beta\left|x-x_{0}\right|^{\beta-1} L\left(\left|x-x_{0}\right|\right)\right. \\
& \left.+\beta(1-\beta)\left|x-x_{0}\right|^{\beta-2} \sum_{i, j=1}^{N} a_{i j} D_{i}\left(\left|x-x_{0}\right|\right) D_{j}\left(\left|x-x_{0}\right|\right)\right) \\
& +R R^{q /(1-q)}\left|x-x_{0}\right|^{\beta q}+g(x) R^{p /(1-q)}\left|x-x_{0}\right|^{\beta p} \\
\geq & R^{1 /(1-q)}\left(\left|x-x_{0}\right|^{\beta q}+\beta\left|x-x_{0}\right|^{\beta-1} L\left(\left|x-x_{0}\right|\right)\right. \\
& \left.+\beta(1-\beta)\left|x-x_{0}\right|^{\beta-2} \sum_{i, j=1}^{N} a_{i j} D_{i}\left(\left|x-x_{0}\right|\right) D_{j}\left(\left|x-x_{0}\right|\right)\right) \geq 0,
\end{aligned}
$$

by (2.6). In $B\left(x_{0}, \delta\right) \backslash B\left(x_{0}, \delta_{0}\right)$, we have that $L\left(\Phi_{2}\right)+R \Phi_{2}^{q}+g(x) \Phi_{2}^{p} \geq 0$. Finally, in $\partial B\left(x_{0}, \delta\right), \Phi$ is bigger than $\gamma$ provided that (2.7) holds.

Hence we can apply [4, Lemma I.1] and conclude that $\Phi$ is a supersolution of (2.5) in $B\left(x_{0}, \delta\right)$. This completes the proof.

For $R>0$, we define the set

$$
N(R):=\left\{x \in \Omega: f^{-}(x) \geq R\right\}=\{x \in \Omega: f(x) \leq-R\},
$$

where $f^{ \pm}(x):=\max \{ \pm f(x), 0\}$. Assume that $f^{ \pm} \not \equiv 0$. The main result of this section is the following theorem.

THEOREM 2.4. Assume that there exists $R>0$ such that

(1) $\delta_{R}:=\left(f_{M} e_{M} / R\right)^{1 /(\beta(1-q))} \leq \delta_{0}$,

(2) $M(R):=\left\{x \in N(R): \operatorname{dist}(x, \partial N(R) \backslash \partial \Omega) \geq \delta_{R}\right\} \neq \emptyset$.

Then there exists a dead core for any nonnegative solution $w$ of (2.2). Moreover, we have $M(R) \subset \Omega_{0}=\{x \in \Omega: w(x)=0\}$.

Proof. Let $x_{0} \in M(R)$, then

$$
\mathscr{B}\left(x_{0}, \delta_{R}\right):=\left\{x \in \Omega:\left|x-x_{0}\right|<\delta_{R}\right\} \subset N(R) .
$$


We call $z$ the unique nonnegative solution of $(2.5)$ in $\mathscr{B}\left(x_{0}, \delta_{R}\right)$ with $\gamma=\left(f_{M} e_{M}\right)^{1 /(1-q)}$. Then by (2.8) we have that

$$
L \theta_{\mid L, q, p, f, g]} \leq-R \theta_{[L, q, p, f, g]}^{q}-g(x) \theta_{[L, q, p, f, g]}^{p} \text { in } \mathscr{B}\left(x_{0}, \delta_{R}\right),
$$

which implies that

$$
L\left(z-\theta_{[L, q, p, f, g]}\right) \geq R\left(\theta_{[L, q, p, f, g]}^{q}-z^{q}\right)+g(x)\left(\theta_{[L, q, p, f, g]}^{p}-z^{p}\right) \quad \text { in } \mathscr{B}\left(x_{0}, \delta_{R}\right),
$$

and by (2.3) and the choice of $\gamma$ we get $z \geq \theta_{[L, q, p, f, g]}$ on $\partial \mathscr{B}\left(x_{0}, \delta_{R}\right)$. Hence, if we denote by $\Omega_{1}:=\left\{x \in \mathscr{B}\left(x_{0}, \delta_{R}\right): z(x)<\theta_{[L, q, p, f, g]}(x)\right\}$,

$$
\begin{aligned}
L\left(z-\theta_{[L, q, p, f, g]}\right) \geq 0 & \text { in } \Omega_{1}, \\
z-\theta_{[L, q, p, f, g]} \geq 0 & \text { on } \partial \Omega_{1} \cap \partial \mathscr{B}\left(x_{0}, \delta_{R}\right), \\
z-\theta_{[L, q, p, f, g]}=0 & \text { on } \partial \Omega_{1} \cap \mathscr{B}\left(x_{0}, \delta_{R}\right) .
\end{aligned}
$$

The maximum principle implies that $z \geq \theta_{[L, q, p, f, g]}$ in $\mathscr{B}\left(x_{0}, \delta_{R}\right)$. Finally, we can apply Lemma 2.3 because $\delta_{R}$ satisfies (2.7). This finishes the proof.

As consequence of the above result, we have the following corollary.

COROLLARY 2.5. Any nonnegative solution of (2.2) has a dead core provided that $f_{M}$ is sufficiently small.

PROOF. It is sufficient to repeat the proof of [13, Remark 2.13] and to take account of the fact that $\delta_{R} \rightarrow 0$ as $f_{M} \rightarrow 0$.

\section{Existence of nonnegative solutions}

Hereafter we write $\theta_{\left[L_{1}, f, g\right]}:=\theta_{\left[L_{1}, 1 / m, 2 / m, f, g\right]}$ and $\theta_{\left[L_{2}, f, g\right]}:=\theta_{\left[L_{2}, 1 / n, 2 / n, f, g\right]}$. The following result gives us a necessary and sufficient condition to obtain semicoexistence states.

THEOREM 3.1. Problem (1.3) has a semicoexistence state if, and only if, $\lambda>0$ and $\mu>0$.

ProOF. By Theorem 2.1 (3) it follows that

$$
u \leq \theta_{\left[L_{1}, \lambda, a\right]} \text { and } v \leq \theta_{\left[L_{2}, \mu, d\right]}
$$

So, if $\lambda \leq 0$, again by Theorem 2.1 (1) we obtain that $u \equiv 0$. Analogously, if $\mu \leq 0, v \equiv 0$. Assume now that $\lambda>0$ and $\mu>0$. In this case, we have that 
$A(x):=\lambda-b(x) \theta_{\left[L_{2}, \mu, d\right]}^{1 / n}(x)$ and $B(x):=\mu-c(x) \theta_{\left[L_{1}, \lambda, a\right]}^{1 / m}(x)$ satisfy $A_{M}=\lambda>0$ and $B_{M}=\mu>0$. We consider the pair

$$
(\underline{u}, \bar{u})=\left(\theta_{\left[L_{1}, A, a\right]}, \theta_{\left[L_{1}, \lambda, a\right]}\right) \quad \text { and } \quad(\underline{v}, \bar{v})=\left(\theta_{\left[L_{2}, B, d\right]}, \theta_{\left.\mid L_{2}, \mu, d\right]}\right) .
$$

By the definitions of $A$ and $B$ and Theorem 2.1 it follows that $\underline{u} \leq \bar{u}, \underline{v} \leq \bar{v}$ and that $\underline{u}$ and $\underline{v}$ are nonnegative and nontrivial functions. Finally, it is not hard to prove that the pair $(\underline{u}, \bar{u})-(\underline{v}, \bar{v})$ is a sub-supersolution of (1.3). This completes the proof.

The following result provides us with conditions which assure the existence of coexistence states as well as their bounds.

THEOREM 3.2. If $\lambda$ and $\mu$ satisfy

$$
\lambda>\left(b(x) \theta_{\left[L_{2, \mu, d]}\right]}^{1 / n}\right)_{M} \quad \text { and } \quad \mu>\left(c(x) \theta_{\left[L_{1, \lambda, a]}\right.}^{1 / m}\right)_{M},
$$

then (1.3) possesses a coexistence state. Moreover, for any coexistence state $(u, v)$ of (1.3) we have the following estimates: if $\lambda>\left(b(x) \theta_{\left[L_{2}, \mu, d\right]}^{1 / n}\right)_{M}$ then

$$
\varepsilon_{1} \varphi_{1} \leq \theta_{\left[L_{1}, A, a\right]} \leq u \leq \theta_{\left[L_{1}, \lambda, a\right]} \leq \lambda^{m /(m-1)}\left(e_{1}\right)_{M}^{1 /(m-1)} e_{1},
$$

and if $\mu>\left(c(x) \theta_{\left[L_{1}, \lambda, a\right]}^{1 / m}\right)_{M}$, then

$$
\varepsilon_{2} \varphi_{2} \leq \theta_{\left[L_{2}, B, d\right]} \leq v \leq \theta_{\left[L_{2}, \mu, d\right]} \leq \mu^{n /(n-1)}\left(e_{2}\right)_{M}^{1 /(n-1)} e_{2},
$$

where $\varphi_{i}$ and $e_{i}, i=1,2$, are the principal positive eigenfunctions of $L_{i}$ and solutions of (2.4) with $L_{i}$ respectively, and $\varepsilon_{1}$ and $\varepsilon_{2}$ are the positive solutions of

$$
\begin{gathered}
\varepsilon_{1}^{1-1 / m} \sigma_{1}\left(L_{1}\right)+a_{M} \varepsilon_{1}^{1 / m}=\lambda-b_{M} \mu^{1 /(n-1)}\left(e_{2}\right)_{M}^{1 /(n-1)}, \\
\varepsilon_{2}^{1-1 / n} \sigma_{1}\left(L_{2}\right)+d_{M} \varepsilon_{1}^{1 / n}=\mu-c_{M} \lambda^{1 /(m-1)}\left(e_{1}\right)_{M}^{1 /(m-1)} .
\end{gathered}
$$

PROoF. Consider the same sub-supersolution as that in the proof of Theorem 3.1. Observe that if (3.2) is satisfied, then $A_{L}>0$ and $B_{L}>0$. Hence Theorem 2.1 (5) completes the existence of a coexistence state.

The estimates (3.1) and (2.3) yield the upper bounds of (3.3) and (3.4). On the other hand, thanks to (3.1), $u$ is a supersolution of (2.2) with $L=L_{1}, f \equiv A$ and $g \equiv a$. So, since $\lambda>\left(b(x) \theta_{\left[L_{2}, \mu, d\right]}^{1 / n}\right)_{M}$ then $A_{L}>0$ and by Theorem $2.1(5)$ the lower bounds of (3.3) follow. Estimates (3.4) can be proved similarly.

REMARK 3. (1) Using (3.3) and (3.4) we can obtain a sufficient condition for the existence of coexistence states involving the coefficients of the problem. Indeed, if $\lambda$ and $\mu$ satisfy

$$
\lambda>b_{M}\left(e_{2}\right)_{M}^{1 /(n-1)} \mu^{1 /(n-1)} \text { and } \mu>c_{M}\left(e_{1}\right)_{M}^{1 /(m-1)} \lambda^{1 /(m-1)},
$$

then $\lambda$ and $\mu$ satisfy (3.2), so that (1.3) has a coexistence state. 

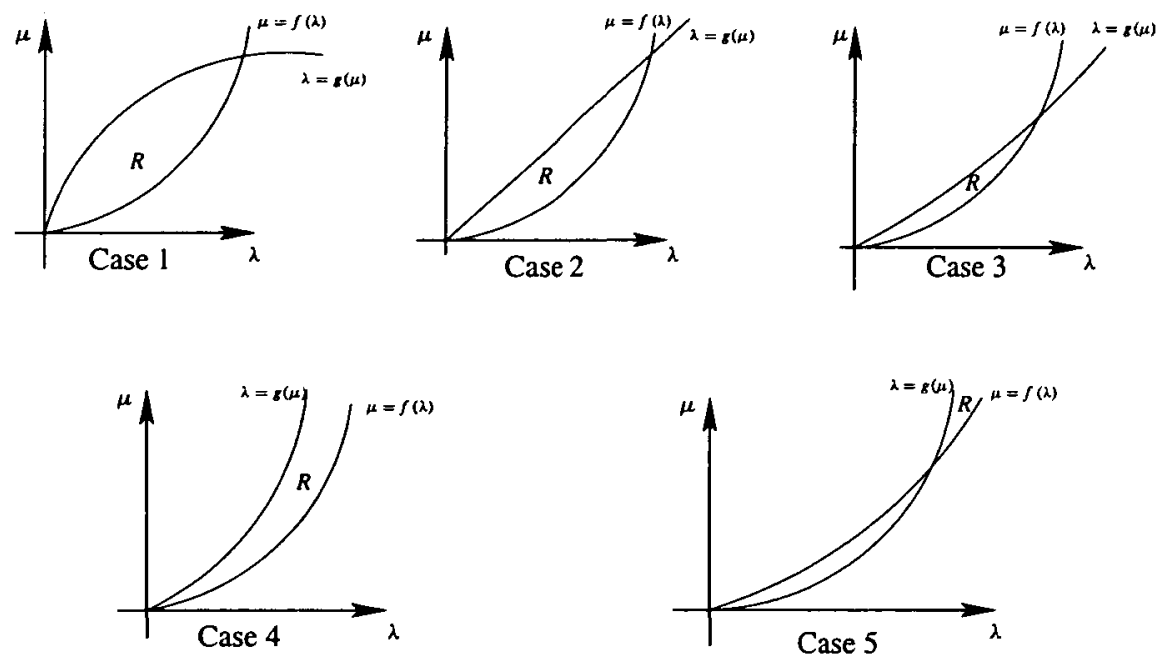

FIGURE 1. $R$ stands for the semicoexistence region in the following cases: Case $1: 1<m, n<2$; Case 2: $1<m<2=n$; Case 3: $1<m<2<n, n-1<1 /(m-1)$; Case 4: $1<m<2<n$, $n-1=1 /(m-1), c_{M} b_{M}^{n-1}\left(e_{1}\right)_{M}^{n-1}\left(e_{2}\right)_{M}^{n-1}<1$; Case 5: $1<m<2<n, n-1>1 /(m-1)$.

(2) Observe that when all coefficients are positive constants (see [28] and [13]) the conditions which assure the existence of coexistence states are independent of $m$ and $n$. This is due to the fact that positive constants are supersolutions of (1.3).

In Figure 1 we have shown the different forms of the region defined in the $(\lambda, \mu)$ plane by (3.6) when $m$ and $n$ vary. We have denoted by $f(\lambda)=c_{M}\left(e_{1}\right)_{M}^{1 /(m-1)} \lambda^{1 /(m-1)}$ and $g(\mu)=b_{M}\left(e_{2}\right)_{M}^{1 /(n-1)} \mu^{1 /(n-1)}$.

3.1. Existence of dead cores We will use the results of Section 2 to show the existence of dead cores for (1.3). The first result provides us with conditions which assure the non-existence of dead cores, and it is a direct consequence of (3.3) and (3.4).

PROPOSITION 3.3. Assume that $\lambda$ and $\mu$ satisfy (3.2). Then any nonnegative solution of (1.3) does not have a dead core.

To state the main result of this section we need some notation. We fix $\lambda>0$. It is not hard to prove that the map $\mu \mapsto \theta_{\left[L_{2}, \mu, d\right]}$ is strictly increasing, and so also is $\mu \mapsto\left(b(x) \theta_{\left[L_{2}, \mu, d\right]}^{1 / n}\right)_{M}$. Hence there exists a unique value $\mu^{*}(\lambda)$ such that $\lambda=\left(b(x) \theta_{\left[L_{2,}, \mu^{*}(\lambda), d\right]}^{1 / n}\right)_{M}$. For such $\lambda$ fixed, we write $\mu_{*}(\lambda)=\left(c(x) \theta_{\left[L_{1}, \lambda, a\right]}^{1 / m}\right)_{M}$.

Analogously, for fixed $\mu>0$, there exists a unique $\lambda^{*}(\mu)>0$ such that $\mu=$ $\left(c(x) \theta_{\left[L_{1}, \lambda^{*}(\mu), a\right]}^{1 / m}\right)_{M}$ and we define $\lambda_{*}(\mu)=\left(b(x) \theta_{\left[L_{2}, \mu, d\right]}^{1 / n}\right)_{M}$. 
ThEOREM 3.4. (1) Assume $\lambda>0$. Then there exist $0<\mu_{1}(\lambda)<\mu_{2}(\lambda)$ with $\mu_{1}(\lambda)<\min \left\{\mu_{*}(\lambda), \mu^{*}(\lambda)\right\}$ and $\max \left\{\mu_{*}(\lambda), \mu^{*}(\lambda)\right\}<\mu_{2}(\lambda)$ such that if $0<\mu<\mu_{1}(\lambda)$ or $\mu>\mu_{2}(\lambda)$ any nonnegative solution of (1.3) has a dead core.

(2) Assume $\mu>0$. Then there exist $0<\lambda_{1}(\mu)<\lambda_{2}(\mu)$ with $\lambda_{1}(\mu)<\min \left\{\lambda_{*}(\mu)\right.$, $\left.\lambda^{*}(\mu)\right\}$ and $\max \left\{\lambda_{*}(\mu), \lambda^{*}(\mu)\right\}<\lambda_{2}(\mu)$ such that if $0<\lambda<\lambda_{1}(\mu)$ or $\lambda>\lambda_{2}(\mu)$ any nonnegative solution of (1.3) has a dead core.

PROOF. We prove (1). The second part follows analogously. Observe that if $\mu<\mu^{*}(\lambda)$ by (3.3) we get

$$
u \geq \theta_{\left[L_{1}, A(x), a\right]} .
$$

Now we define $F(x, \mu):=\mu-a(x, \mu):=\mu-c(x) \theta_{\left[L_{1}, A(x), a\right]}^{1 / m}$. Using (3.7) it is not hard to prove that $v$ is a subsolution of (2.2) with $L=L_{2}, f(x)=F(x, \mu)$ and $g(x)=d(x)$, and so by Theorem 2.1 (3), it follows that

$$
v \leq \theta_{\left[L_{2}, F(x, \mu), d\right]} .
$$

Now we are going to use Theorem 2.4 to prove that $\theta_{\left[L_{2}, F(x, \mu), d\right]}$ has a dead core, so that by (3.8) the result follows.

Observe that in this case $(F(x, \mu))_{M}=\mu$ and so $\delta_{R}=\left(\mu\left(e_{2}\right)_{M} / R\right)^{n /(\beta(n-1))}$. On the other hand, since $(a(x, 0))_{M}>0$ and $(a(x, \mu))_{M}$ is decreasing in $\mu$, there exists a unique $\mu_{0}(\lambda)>0$ such that $\mu_{0}=\left(a\left(x, \mu_{0}\right)\right)_{M}$. Observe that, by the definition of $\mu_{*}(\lambda)$, we have that $\mu_{0}(\lambda)<\mu_{*}(\lambda)$. Taking $\mu \leq \mu_{0} / 2$, we have $0<\mu \leq \mu_{0} / 2<$ $\mu_{0}=\left(a\left(x, \mu_{0}\right)\right)_{M}<(a(x, \mu))_{M}$. Hence there exists $R_{0}>0$ such that the following set is nonempty:

$$
\left\{x \in \Omega: \mu_{0}(\lambda) / 2-a\left(x, \mu_{0}(\lambda) / 2\right) \leq-R_{0}\right\} \neq \emptyset .
$$

We define $N(\mu):=N\left(R_{0}\right)=\left\{x \in \Omega: F(x, \mu) \leq-R_{0}\right\}$. Again by Theorem 2.1 we get that if $\mu_{1} \leq \mu_{2}$ then $F\left(x, \mu_{1}\right) \leq F\left(x, \mu_{2}\right)$. Hence if $\mu_{1} \leq \mu_{2} \leq \mu_{0} / 2$ then

$$
\emptyset \neq N\left(\mu_{0} / 2\right) \subset N\left(\mu_{2}\right) \subset N\left(\mu_{1}\right) .
$$

Let $x_{0} \in \Omega$ be the point where $F\left(x, \mu_{0} / 2\right)$ attains its negative minimum. For that $x_{0} \in \Omega$, there exists $r_{0}>0$ such that $B\left(x_{0}, r_{0}\right) \subset N\left(\mu_{0} / 2\right)$.

Finally, since $\delta_{R_{0}} \rightarrow 0$ as $\mu \rightarrow 0$ there exists $\mu^{\prime}(\lambda)>0$ such that for $\mu<\mu^{\prime}(\lambda)$, $\delta_{R_{0}}<\min \left\{r_{0}, \delta_{0}\right\}$. Define $\mu_{1}(\lambda):=\min \left\{\mu_{*}(\lambda), \mu^{*}(\lambda), \mu_{0} / 2(\lambda), \mu^{\prime}(\lambda)\right\}$ and therefore for $\mu<\mu_{1}(\lambda)$ we get

$$
\operatorname{dist}\left(x_{0}, \partial N(\mu) \backslash \partial \Omega\right) \geq \operatorname{dist}\left(x_{0}, \partial N\left(\mu_{0} / 2\right) \backslash \partial \Omega\right) \geq \operatorname{dist}\left(x_{0}, \partial B\left(\dot{x}_{0}, r_{0}\right)\right)>\delta_{R_{0}} .
$$

Therefore $M(\mu) \neq \emptyset$ and Theorem 2.4 completes the first part of (1). 
For the second part, take $\mu>\max \left\{\mu_{*}(\lambda), \mu^{*}(\lambda)\right\}$, so $\mu>\left(c(x) \theta_{\left[L_{1}, \lambda, a\right]}^{1 / m}\right)_{M}$ and by (3.4) we get that $v \geq \theta_{\left[L_{2}, B(x), d\right]}$. Now we define

$$
G(x, \mu):=\lambda-b(x, \mu):=\lambda-b(x) \theta_{\left[L_{2}, B(x), d\right]}^{1 / n} .
$$

With a similar reasoning to that used in (3.8) we get that $u \leq \theta_{\left[L_{1}, G(x, \mu), a\right]}$. In this case, we take $R=\mu^{r}$ with $r>0$ to be chosen later. So

$$
N(R)=N(\mu):=\left\{x \in \Omega: \lambda \leq b(x, \mu)-\mu^{r}\right\}
$$

and

$$
(G(x, \mu))_{M}=\lambda, \quad \delta_{R}=\left(\lambda\left(e_{1}\right)_{M} / \mu^{r}\right)^{m /(\beta(m-1))} .
$$

Using (3.4), we have that $b(x, \mu) \geq b(x) \varepsilon_{2}^{1 / n}(\mu) \varphi_{2}^{1 / n}$, where $\varepsilon_{2}$ is defined in (3.5).

Let $\delta>0$ be sufficiently small such that

$$
B_{\delta}^{+}:=\left\{x \in B^{+}: \operatorname{dist}\left(x, \partial B^{+}\right) \geq \delta\right\} \neq \emptyset,
$$

where $B^{+}:=\{x \in \Omega: b(x)>0\}$. Define the set

$$
T(\mu):=\left\{x \in B_{\delta}^{+}: \lambda \leq \varepsilon_{2}^{1 / n}(\mu) b(x) \varphi_{2}^{1 / n}(x)-\mu^{r}\right\} .
$$

Clearly, $T(\mu) \subset N(\mu)$.

On the other hand, by Remark $1, \varepsilon_{2}^{1 / n}(\mu)=O\left(\mu^{1 /(n-1)}\right)$ if $n \geq 2$ and $\varepsilon_{2}^{1 / n}(\mu)=$ $O(\mu)$ if $n<2$ when $\mu$ is large. Take $r<1 /(n-1)$. if $n \geq 2$ and $r<1$ if $n<2$. So there exists $\mu^{0}(\lambda)>0$ such that for $\mu>\mu^{0}(\lambda)$,

$$
T\left(\mu^{0}(\lambda)\right) \neq \emptyset \quad \text { and } \quad T\left(\mu^{0}(\lambda)\right) \subset T(\mu) .
$$

Moreover, there exist $x_{0} \in B_{\delta}^{+}$and $r_{0}>0$ such that

$$
B\left(x_{0}, r_{0}\right) \subset T\left(\mu_{0}(\lambda)\right) \text {. }
$$

Furthermore, since $\delta_{R} \rightarrow 0$ as $\mu \rightarrow \infty$, there exists $\mu^{\prime \prime}(\lambda)$ such that for $\mu>$ $\mu^{\prime \prime}(\lambda)$ we get $\delta_{R}<\min \left\{r_{0}, \delta_{0}\right\}$. Hence, using (3.9) and (3.10), for $\mu>\mu_{2}(\lambda):=$ $\max \left\{\mu_{*}(\lambda), \mu^{*}(\lambda), \mu^{0}(\lambda), \mu^{\prime \prime}(\lambda)\right\}$, we obtain

$$
\begin{aligned}
\operatorname{dist}\left(x_{0}, \partial N(\mu) \backslash \partial \Omega\right) & \geq \operatorname{dist}\left(x_{0}, \partial T(\mu)\right) \geq \operatorname{dist}\left(x_{0}, \partial T\left(\mu^{0}\right)\right) \\
& \geq \operatorname{dist}\left(x_{0}, \partial B\left(x_{0}, r_{0}\right)\right)=r_{0}>\delta_{R} .
\end{aligned}
$$

Theorem 2.4 completes the proof.

REMARK 4. (1) By the proof of Theorem 3.4, we can see that if $\mu<\mu_{1}(\lambda)$ (or $\left.\lambda>\lambda_{2}(\mu)\right)$ for any semicoexistence state $(u, v)$ then $v$ has a dead core. Similarly, if $\mu>\mu_{2}(\lambda)$ (or $\lambda<\lambda_{1}(\mu)$ ) then $u$ has a dead core. 
(2) We can give a biological interpretation of Theorem 3.4. If we fix the growth rates of $u$ and $\lambda$, then the other species does not live in all its habitat if its growth rate is small. But, if the growth rate of $v$ is large, then $u$ cannot survive in all of $\Omega$.

On the other hand, when $m=n=1$ it was shown in [21] (see also [27]) that if the interaction rate (for example) $b$ is large, then $v$ drives $u$ to extinction. This is in strong contrast with the case when $m, n>1$, because by Theorem 3.1 neither species drives the other to extinction when $b$ or $c$ is large.

\section{Maximum principle for a singular system}

We define in $X^{2}$ the following order: given $\left(u_{1}, v_{1}\right),\left(u_{2}, v_{2}\right) \in X^{2}$,

$$
\left(u_{1}, v_{1}\right) \leq\left(u_{2}, v_{2}\right) \text { if and only if } u_{1} \leq u_{2} \text { and } v_{1} \geq v_{2} \text {. }
$$

Analogously, we write $\left(u_{1}, v_{1}\right) \prec\left(u_{2}, v_{2}\right)$ if $u_{1}<u_{2}$ and $v_{1} \geq v_{2}$ or $u_{1} \leq u_{2}$ and $v_{1}>v_{2}$.

Let $M(x)=\left(m_{i j}(x)\right)$ be a $2 \times 2$ matrix whose elements belong to the Fréchet space $C^{1}(\Omega)$ and such that there exist $K>0$ and $\alpha \in(0,2]$ satisfying:

(HM) $m_{i j} \geq 0, m_{i j} \neq \equiv, i \neq j$;

$$
\left|m_{i j}(x)\right| \operatorname{dist}(x, \partial \Omega)^{2-\alpha} \leq K \quad i, j=1,2 .
$$

The object of this section is to analyse the following singular eigenvalue problem:

$$
\begin{cases}\mathscr{L} U+M(x) U=\sigma U & \text { in } \Omega, \\ U=0 & \text { on } \partial \Omega\end{cases}
$$

where $\mathscr{L}=\operatorname{diag}\left(L_{1}, L_{2}\right), U=(u, v)^{t}$, and $L_{i}, i=1,2$, are operators as in (1.2).

The next result characterises the existence of a positive eigenvalue of (4.2) by means of the existence of a strict positive supersolution in the following sense. The proof of the result follows from [27, Theorem 6.3] and [15, Section 2].

Definition 4.1. We say that $\Phi \in\left(C^{2}(\Omega) \cap C^{1, \delta}(\bar{\Omega})\right)^{2}, \delta \in(0,1), \Phi \succ 0$ is a supersolution of $\mathscr{L}+M$ if $(\mathscr{L}+M) \Phi \succeq 0$ in $\Omega$ and $\Phi \succeq 0$ on $\partial \Omega$. If in addition, $(\mathscr{L}+M) \Phi \succ 0$ in $\Omega$ or $\Phi \succ 0$ on $\partial \Omega$, then it is said that $\Phi$ is a strict supersolution.

THEOREM 4.2. Under the assumption (HM), the following conditions are equivalent:

(1) $\mathscr{L}+M$ admits a positive strict supersolution;

(2) The operator $[\mathscr{L}+M]^{-1}: X^{2} \mapsto X^{2}$ is well defined, compact and strongly positive; 
(3) The problem

$$
\begin{cases}\mathscr{L} U+M(x) U=F & \text { in } \Omega \\ U=0 & \text { on } \partial \Omega\end{cases}
$$

where $F \in Y^{2}$, satisfies the strong maximum principle, that is, if $F \succeq 0$ and $F \neq 0$, then $U \succ 0$;

(4) The operator $[\mathscr{L}+M]: X^{2} \mapsto Y^{2}$ possesses a strictly positive eigenvalue, denoted by $\sigma_{1}(\mathscr{L}+M)$. This eigenvalue is simple and it is the only eigenvalue of (4.2) possessing a positive eigenfunction $\Phi_{1} \succ 0$.

In the present work, we need to apply this result assuming less regularity for the strict supersolution.

Proposition 4.3. Assume that $M$ satisfies (HM). Then $\sigma_{1}(\mathscr{L}+M)>0$ if and only if there exists $\Phi \in\left(C^{2}(\Omega) \cap C_{0}^{0}(\bar{\Omega})\right)^{2}$ such that $\Phi \succ 0$ in $\Omega$ and $(\mathscr{L}+M) \Phi \succ 0$ in $\Omega$.

REMARK 5. Since $\Phi=\left(\Phi_{1}, \Phi_{2}\right) \notin X^{2}$, when we write $\Phi \succ 0$ we mean that $\Phi_{1}(x)>0$ and $\Phi_{2}(x)<0$ for all $x \in \Omega$.

The following boundary point result will be used in the proof.

LEMMA 4.4. Let $u \in C^{2}(\Omega) \cap C^{1}(\bar{\Omega})$ be such that $u \geq 0$ in $\Omega, u \neq 0$ and

$$
(L+q) u \geq 0 \text { in } \Omega, \quad u \geq 0 \text { on } \partial \Omega,
$$

where $q \in C^{1}(\Omega)$ satisfies (4.1). Then $u(x)>0$ for all $x \in \Omega$ and for all $x_{0} \in \partial \Omega$ such that $u\left(x_{0}\right)=0,(\partial u / \partial n)\left(x_{0}\right)<0$.

Proof. It is an easy consequence of [5, Lemma 3.6] with $\rho(r)=r^{\alpha-2}$.

Proof of Proposition 4.3. It is clear that if $\sigma_{1}(\mathscr{L}+M)>0$, we can take $\Phi=\Phi_{1}$, the eigenfunction associated with $\sigma_{1}(\mathscr{L}+M)$.

Now assume that there exists $\Phi \in\left(C^{2}(\Omega) \cap C_{0}^{0}(\bar{\Omega})\right)^{2}$ such that $\Phi>0$ in $\Omega$ and $(\mathscr{L}+M) \Phi:=G \succ 0$ in $\Omega$. Let $F \succeq 0$ and $F \not \equiv 0$ and $U$ be the solution of (4.3). We have to prove that $U>0$ and then, by Theorem 4.2 , the proof is concluded.

For each $\varepsilon>0$ and $K>0$, we define

$$
W:=U+(\varepsilon,-\varepsilon)^{t}+\varepsilon K \Phi \in\left(C^{2}(\Omega) \cap C^{0}(\bar{\Omega})\right)^{2} .
$$

Since $\Phi \in\left(C_{0}^{0}(\bar{\Omega})\right)^{2}$, for any $\varepsilon>0$, there exists $\gamma(\varepsilon)>0$ such that $W \succ 0$ in $\Omega_{\varepsilon}:=\{x \in \Omega: \operatorname{dist}(x, \partial \Omega)<\gamma(\varepsilon)\}$. Moreover

$$
(\mathscr{L}+M) W \succeq \varepsilon\left(\left(m_{11}-m_{12}, m_{21}-m_{22}\right)^{t}+K G\right) \succ 0 \quad \text { in } \Omega \backslash \bar{\Omega}_{\varepsilon},
$$


for $K$ sufficiently large. Now in $\Omega \backslash \bar{\Omega}_{\varepsilon}$ the coefficients $m_{i j}$ are bounded. So, since $\Phi$ is a strict supersolution in $\Omega \backslash \bar{\Omega}_{\varepsilon}$, we can apply [27, Theorem 6.3] to get that $W \succ 0$ in $\Omega \backslash \bar{\Omega}_{\varepsilon}$. Thus $W>0$ in $\Omega$ for all $\varepsilon>0$, and we obtain that $U \succeq 0$ in $\Omega$. Let $U=\left(u_{1}, u_{2}\right)^{\prime}$. Since $U \neq(0,0)$ we can assume that $u_{1} \geq 0$ and $u_{1} \not \equiv 0$. Then, denoting $F=\left(f_{1}, f_{2}\right)^{t}$ and taking into account that $m_{12} \geq 0$, we obtain

$$
L_{1} u_{1}+m_{11} u_{1}=f_{1}-m_{12} u_{2} \geq 0 \text { in } \Omega, \quad u_{1}=0 \text { on } \partial \Omega,
$$

and so applying Lemma 4.4, we get $u_{1}>0$. For the second equation,

$$
L_{2}\left(-u_{2}\right)+m_{22}\left(-u_{2}\right)=-f_{2}+m_{21} u_{1}>0 \text { in } \Omega, \quad u_{2}=0 \text { on } \partial \Omega
$$

and so $-u_{2}>0$. So $U \succ 0$. This completes the proof.

Again, the next result is a consequence of [27, Theorem 6.5] and [15, Theorem 4].

THEOREM 4.5. Assume (HM) holds. There exists one real eigenvalue of (4.2), denoted $\sigma_{1}(\mathscr{L}+M)$, associated with a positive eigenfunction $\Phi_{1} \succ 0$. The eigenvalue is simple and there is no other eigenvalue associated with a positive eigenfunction.

The following result will be used to compare principal eigenvalues of different matrices.

LEMMA 4.6. Let $A(x)=\left(a_{i j}(x)\right)$ and $B(x)=\left(b_{i j}(x)\right)$ be two matrices with $a_{i j}, b_{i j}$ satisfying $(H M), b_{i i} \geq a_{i i}$ and $a_{i j} \geq b_{j i}$ for $i \neq j$ with some inequality strict. Then $\sigma_{1}(\mathscr{L}+A)<\sigma_{1}(\mathscr{L}+B)$.

PROOF. Let $\Phi^{A} \succ 0$ be the eigenfunction associated with $\mathscr{L}+A$. Then it is easy to show that $\left(\mathscr{L}+B-\sigma_{1}(\mathscr{L}+A) I\right) \Phi^{A} \succ 0$, and so $\Phi^{A}$ is a strict supersolution of $\mathscr{L}+B-\sigma_{1}(\mathscr{L}+A) I$. Hence, by Theorem 4.2, we deduce that

$$
\sigma_{1}\left(\mathscr{L}+B-\sigma_{1}(\mathscr{L}+A) I\right)>0
$$

whence the conclusion follows.

\section{Uniqueness result}

Throughout this section we assume that $\lambda$ and $\mu$ satisfy (3.2), and so the validity of the strong maximum principle is guaranteed. Indeed, by (3.4) we get

$$
u^{1 / m}\left(\lambda-b(x) v^{1 / n}\right)-a(x) u^{2 / m} \geq u^{1 / m}\left(\lambda-b(x) \theta_{\left[L_{2, \mu, d]}\right)}^{1 / n}-a(x) u^{2 / m},\right.
$$


and so, by (3.2), there exists a positive constant $M$ such that

$$
u^{1 / m}\left(\lambda-b(x) v^{1 / n}\right)-a(x) u^{2 / m}+M u \geq 0,
$$

whence it follows that if $(u, v)$ is a nonnegative solution of (1.3) with $u \not \equiv 0$, then $u(x)>0$ for all $x \in \Omega$. Similarly we can reason with the second equation in (1.3).

In this section we obtain a uniqueness result for a coexistence state of (1.3). In order to get the result we use the fixed point index in cones.

For the fixed $M>0$ obtained in (5.1), consider the operator $\mathscr{K}: X^{2} \mapsto X^{2}$ defined by

$$
\mathscr{K}(u, v):=\left(\begin{array}{c}
\left(L_{1}+M\right)^{-1}\left(u^{1 / m}\left(\lambda-a(x) u^{1 / m}-b(x) v^{1 / n}\right)+M u\right) \\
\left(L_{2}+M\right)^{-1}\left(v^{1 / n}\left(\mu-d(x) v^{1 / n}-c(x) u^{1 / m}\right)+M v\right)
\end{array}\right)
$$

where $\left(L_{i}+M\right)^{-1}, i=1,2$, stands for the inverse on the operator $L_{i}+M$ in $\Omega$ under homogeneous Dirichlet boundary conditions. Observe that by $(2.1), \sigma_{1}\left(L_{i}+M\right)>0$ and so $\left(L_{i}+M\right)^{-1}$ is well defined and is a compact operator. Thanks to the choice of $M$, see (5.1), $\mathscr{K}$ is a positive operator whose fixed points are componentwise nonnegative solutions of (1.3).

On the other hand, by (3.3) and (3.4), there exist $R_{i}>0, i=1,2$, such that for every $(u, v)$ coexistence states of (1.3),

$$
\|u\|_{\infty} \leq R_{1}:=\left(\lambda\left(e_{1}\right)_{M}\right)^{m /(m-1)} \quad \text { and } \quad\|v\|_{\infty} \leq R_{2}:=\left(\mu\left(e_{2}\right)_{M}\right)^{n /(n-1)} .
$$

So the fixed point index of $\mathscr{K}$ over $\mathscr{B}$ with respect to the cone $P \times P$ is well defined, where $\mathscr{B}:=\left\{(u, v) \in P^{2}:\|u\|_{\infty} \leq R_{1}+1,\|v\|_{\infty} \leq R_{2}+1\right\}$. We are now going to compute this index in some cases.

Proposition 5.1. Assume that $\lambda$ and $\mu$ satisfy (3.2). The following assertions are true:

(1) $i_{P \times P}(\mathscr{H}, \mathscr{B})=1$;

(2) $i_{P \times P}(\mathscr{K},(0,0))=0$;

(3) $i_{P \times P}\left(\mathscr{K},\left(\theta_{\left[L_{1}, \lambda, a\right]}, 0\right)\right)=i_{P \times P}\left(\mathscr{K},\left(0, \theta_{\left[L_{2}, \mu, d\right]}\right)\right)=0$.

PROOF. (1) First, we define $\mathscr{G}_{1}: X \mapsto X$ by

$$
\mathscr{G}_{1}(u):=\left(L_{1}+M\right)^{-1}\left(u^{1 / m}\left(\lambda-a(x) u^{1 / m}\right)+M u\right) .
$$

By (2.3), taking $B_{u}:=\left\{u \in P:\|u\|_{\infty} \leq R_{1}+1\right\}$ the fixed point index of $\mathscr{G}_{1}$ over $B_{u}$ is well defined. Applying [2, Lemma 12.1] it can be proved that

$$
i_{P}\left(\mathscr{G}_{1}, B_{u}\right)=1 \text {. }
$$


Indeed, if there exist $t \geq 1$ and $u \in P$ such that $\|u\|_{\infty}=R_{1}+1$ and $\mathscr{G}_{1}(u)=t u$, then

$$
L_{1} u \leq u^{1 / m}\left(\frac{\lambda}{t}-\frac{a(x)}{t} u^{1 / m}\right)
$$

and so $\|u\|_{\infty} \leq(\lambda / t)^{m /(m-1)}\left(e_{1}\right)_{M}^{m /(m-1)} \leq R_{1}<R_{1}+1$. Analogously,

$$
i_{P}\left(\mathscr{G}_{2}, B_{v}\right)=1
$$

with $\mathscr{G}_{2}(v):=\left(L_{2}+M\right)^{-1}\left(v^{1 / n}\left(\mu-d(x) v^{1 / n}\right)+M v\right)$ and $B_{v}:=\left\{v \in P:\|v\|_{\infty} \leq\right.$ $\left.R_{2}+1\right\}$.

Consider the operator $\mathscr{H}_{1}:[0,1] \times X^{2} \mapsto X^{2}$ defined by

$$
\mathscr{H}_{1}(t, u, v):=\left(\begin{array}{l}
\left(L_{1}+M\right)^{-1}\left(u^{1 / m}\left(\lambda-a(x) u^{1 / m}-t b(x) v^{1 / n}\right)+M u\right) \\
\left(L_{2}+M\right)^{-1}\left(v^{1 / n}\left(\mu-d(x) v^{1 / n}-t c(x) u^{1 / m}\right)+M v\right)
\end{array}\right) .
$$

Observe that by (3.3) and (3.4) any fixed point of $\mathscr{H}_{1}$ belongs to $\mathscr{B}$. So, it follows by homotopy invariance, (5.2) and (5.3) that

$$
\begin{aligned}
i_{P \times P}(\mathscr{K}, \mathscr{B}) & =i_{P \times P}\left(\mathscr{H}_{1}(1, \cdot), \mathscr{B}\right)=i_{P \times P}\left(\mathscr{H}_{1}(0, \cdot), \mathscr{B}\right) \\
& =i_{P}\left(\mathscr{G}_{1}, B_{u}\right) \cdot i_{P}\left(\mathscr{G}_{2}, B_{v}\right)=1 .
\end{aligned}
$$

We now prove (2). Let $\psi_{i} \in Y, i=1,2$, be such that $\psi_{i}>0$ in $\Omega$. We define

$$
\mathscr{H}_{2}(t, u, v):=\left(\begin{array}{l}
\left(L_{1}+M\right)^{-1}\left(u^{1 / m}\left(\lambda-a(x) u^{1 / m}-b(x) v^{1 / n}\right)+M u+t \psi_{1}\right) \\
\left(L_{2}+M\right)^{-1}\left(v^{1 / n}\left(\mu-d(x) v^{1 / n}-c(x) u^{1 / m}\right)+M v+t \psi_{2}\right)
\end{array}\right) .
$$

We claim that there exists $\delta>0$ such that

$$
(u, v) \neq \mathscr{H}_{2}(t, u, v), \quad \forall t \in[0,1], \forall(u, v) \in \mathscr{N}_{\delta},
$$

where $\mathscr{N}_{\delta}:=\left\{(u, v) \in P^{2}:\|u\|_{\infty} \leq \delta,\|v\|_{\infty} \leq \delta\right\} \backslash\{(0,0)\}$. Assume there exist sequences $\left(u_{r}, v_{r}\right)$ of functions and $t_{r} \in[0,1]$ such that $\left(u_{r}, v_{r}\right) \rightarrow(0,0)$ as $r \rightarrow \infty$ and $\left(u_{r}, v_{r}\right)=\mathscr{H}_{2}\left(t_{r}, u_{r}, v_{r}\right)$. Since $\lambda>0$ and $\left\|v_{r}\right\|_{\infty} \rightarrow 0$, there exists $r_{0} \in \mathbb{N}$ such that $\left(\lambda-b(x) v_{r}^{1 / n}\right)_{L}>0$ for $r \geq r_{0}$. So, the strong maximum principle is satisfied in the first equation, and so $u_{r}>0$. Let $K>0$ be such that $K \geq \sigma_{1}\left(L_{1}\right)$. Since $\left\|u_{r}\right\|_{\infty} \rightarrow 0$, there exists $r_{1} \in \mathbb{N}$ such that for $r \geq r_{1}$ we have

$$
L_{1} u_{r}=u_{r}^{1 / m}\left(\lambda-b(x) v_{r}^{1 / n}\right)-a(x) u_{r}^{2 / m}+t_{r} \psi_{1}>K u_{r},
$$

and hence $\sigma_{1}\left(L_{1}-K\right)>0$, a contradiction.

Thus by (5.4) the homotopy is admissible and we get

$$
\begin{aligned}
i_{P \times P}(\mathscr{K},(0,0)) & =i_{P \times P}\left(\mathscr{K}, \mathscr{N}_{\delta}\right)=i_{P \times P}\left(\mathscr{H}_{2}(0, \cdot), \mathscr{N}_{\delta}\right) \\
& =i_{P \times P}\left(\mathscr{H}_{2}(1, \cdot), \mathscr{N}_{\delta}\right)=0
\end{aligned}
$$


this last equality following by (5.4).

It remains to prove (3). Let $\psi \in Y$ be such that $\psi>0$ in $\Omega$. We define another operator

$$
\mathscr{H}_{3}(t, u, v):=\left(\begin{array}{c}
\left(L_{1}+M\right)^{-1}\left(u^{1 / m}\left(\lambda-a(x) u^{1 / m}-b(x) v^{1 / n}\right)+M u\right) \\
\left(L_{2}+M\right)^{-1}\left(v^{1 / n}\left(\mu-d(x) v^{1 / n}-c(x) u^{1 / m}\right)+M v+t \psi\right)
\end{array}\right) .
$$

We claim that there exists $\delta>0$ such that

$$
(u, v) \neq \mathscr{H}_{3}(t, u, v), \quad \forall t \in[0,1], \forall(u, v) \in \mathscr{M}_{\delta},
$$

where $\mathscr{M}_{\delta}:=\left\{(u, v) \in P^{2}:\left\|u-\theta_{\left|L_{1}, \lambda, a\right|}\right\|_{\infty} \leq \delta,\|v\|_{\infty} \leq \delta\right\} \backslash\left\{\left(\theta_{\left[L_{1}, \lambda, a\right]}, 0\right)\right\}$. Assume there exist sequences $\left(u_{r}, v_{r}\right) \rightarrow\left(\theta_{\left[L_{1}, \lambda, a\right]}, 0\right)$ as $r \rightarrow \infty$ and $t_{r} \in[0,1]$ such that

$$
\left(u_{r}, v_{r}\right)=\mathscr{H}_{3}\left(t_{r}, u_{r}, v_{r}\right)
$$

Since $u_{r} \leq \theta_{\left[L_{1}, \lambda, a \mid\right.}$ and $\mu>\left(c(x) \theta_{\left[L_{1}, \lambda, a\right]}^{1 / m}\right)_{M}$, it follows that

$$
\mu-c(x) u_{r}^{1 / m}(x) \geq \mu-c(x) \theta_{\left[L_{1}, \lambda, a\right]}^{1 / m}>0
$$

Let $K>0$ be such that $K \geq \sigma_{1}\left(L_{2}\right)$. Then by (5.6) there exists $r_{0} \in \mathbb{N}$ such that for $r \geq r_{0}$ we have

$$
L_{2} v_{r}=v_{r}^{1 / n}\left(\mu-c(x) u_{r}^{1 / m}\right)-d(x) v_{r}^{2 / n}+t_{r} \psi>K v_{r}, \quad \text { in } \Omega
$$

and hence $\sigma_{1}\left(L_{2}-K\right)>0$, a contradiction.

Thus by (5.5) the homotopy is admissible and we get

$$
\begin{aligned}
i_{P \times P}\left(\mathscr{K},\left(\theta_{\left[L_{1}, \lambda, a\right]}, 0\right)\right) & =i_{P \times P}\left(\mathscr{K}, \mathscr{M}_{\delta}\right)=i_{P \times P}\left(\mathscr{H}_{3}(0, \cdot), \mathscr{M}_{\delta}\right) \\
& =i_{P \times P}\left(\mathscr{H}_{3}(1, \cdot), \mathscr{M}_{\delta}\right)=0 .
\end{aligned}
$$

Analogously, it can be treated as the solution $\left(0, \theta_{\left[L_{2}, \mu, d\right]}\right)$.

Let $\left(u_{0}, v_{0}\right)$ be a coexistence state of (1.3). We consider the matrix $M_{\left(u_{0}, v_{0}\right)}:=\left(m_{i j}\right)$, $i, j=1,2$, which is related to the linearisation of (1.3) about $\left(u_{0}, v_{0}\right)$, where

$$
\begin{array}{ll}
m_{11}=-\frac{1}{m} u_{0}^{1 / m-1}\left(\lambda-2 a(x) u_{0}^{1 / m}-b(x) v_{0}^{1 / n}\right), & m_{12}=\frac{1}{n} b(x) u_{0}^{1 / m} v_{0}^{1 / n-1}, \\
m_{22}=-\frac{1}{n} v_{0}^{1 / n-1}\left(\mu-2 d(x) v_{0}^{1 / n}-c(x) u_{0}^{1 / m}\right), & m_{21}=\frac{1}{m} c(x) v_{0}^{1 / n} u_{0}^{1 / m-1} .
\end{array}
$$

Observe that since $\left(u_{0}, v_{0}\right)$ is a coexistence state, by (3.3) and (3.4) there exists $k_{0}>0$ such that $k_{0} \operatorname{dist}(x, \partial \Omega) \leq u_{0}$ and $k_{0} \operatorname{dist}(x, \partial \Omega) \leq v_{0}$, then $M_{\left(u_{0}, v_{0}\right)}$ satisfies $(\mathrm{HM})$, so that $\sigma_{1}\left(\mathscr{L}+M_{\left(u_{0}, \nu_{0}\right)}\right)$ makes sense.

The general uniqueness result reads as follows. 
THEOREM 5.2. Assume that $\lambda$ and $\mu$ satisfy (3.2) and $\sigma_{1}\left(\mathscr{L}+M_{\left(u_{0} . w_{0}\right)}\right)>0$ for any $\left(u_{0}, v_{0}\right)$ coexistence state of (1.3). Then (1.3) possesses a unique coexistence state.

Proof. Recall that by Proposition 3.3, if $\lambda$ and $\mu$ satisfy (3.2) then any nonnegative solution of (1.3) is a coexistence state. We claim that if $\left(u_{0}, v_{0}\right)$ is a coexistence state of (1.3), then

$$
i_{P \times P}\left(\mathscr{K},\left(u_{0}, v_{0}\right)\right)=1
$$

Assume that we have proved (5.8), then since $\mathscr{K}$ is a compact operator, it possesses a finite number of coexistence states, say $\left(u_{i}, v_{i}\right), i=1, \ldots, r$. Then

$$
\begin{aligned}
i_{P \times P}(\mathscr{K}, \mathscr{B})= & i_{P \times P}(\mathscr{K},(0,0))+i_{P \times P}\left(\mathscr{K},\left(\theta_{\left.\mid L_{1}, \lambda, a\right]}, 0\right)\right) \\
& +i_{P \times P}\left(\mathscr{K},\left(0, \theta_{\left[L_{2}, \mu, d\right]}\right)\right)+\sum_{i=1}^{r} i_{P \times P}\left(\mathscr{K},\left(u_{i}, v_{i}\right)\right)
\end{aligned}
$$

and so, by Proposition 5.1 and (5.8), $1=0+0+0+r$, whence the conclusion now easily follows.

It remains to prove (5.8). Let $h \in C^{1}(\Omega)$ be such that $h$ verifies that, for some $\alpha \in(0,2]$ and $K>0,|h(x)| \operatorname{dist}(x, \partial \Omega)^{2-\alpha} \leq K$ and

$$
h \geq \max \left\{0, m_{11}, m_{22}\right\}
$$

where $m_{11}$ and $m_{22}$ are defined in (5.7). We define the operator

$$
\mathscr{T}(u, v):=\left(\begin{array}{l}
\left(L_{1}+h\right)^{-1}\left(u^{1 / m}\left(\lambda-a(x) u^{1 / m}-b(x) v^{1 / n}\right)+h u\right) \\
\left(L_{2}+h\right)^{-1}\left(v^{1 / n}\left(\mu-d(x) v^{1 / n}-c(x) u^{1 / m}\right)+h v\right)
\end{array}\right)
$$

Observe that $\left(L_{i}+h\right)^{-1}$ exists because $h \geq 0$ and so $\sigma_{1}\left(L_{i}+h\right)>0$.

By the Leray-Schauder formula, $i_{P \times P}\left(\mathscr{T},\left(u_{0}, v_{0}\right)\right)=(-1)^{\xi}$, where $\xi$ is the sum of the multiplicities of the eigenvalues of $D_{(u, v)} \mathscr{T}\left(u_{0}, v_{0}\right)$ larger than one, being $D_{(u, v)} \mathscr{T}\left(u_{0}, v_{0}\right)$, the linearisation of $\mathscr{T}$ about $\left(u_{0}, v_{0}\right)$. It is clear that

$$
D_{(u, v)} \mathscr{T}\left(u_{0}, v_{0}\right)=\operatorname{diag}\left(\left(L_{1}+h\right)^{-1},\left(L_{2}+h\right)^{-1}\right)\left(-M_{\left(u_{0}, v_{0}\right)}+\operatorname{diag}(h, h)\right),
$$

where $M_{\left(u_{0}, \omega_{0}\right)}$ is defined by (5.7). It is not hard to prove that if $r>1$ is an eigenvalue of $D_{(u, v)} \mathscr{T}\left(u_{0}, v_{0}\right)$, then

$$
\sigma_{1}\left(\mathscr{L}+M_{\left(u_{0}, \nu_{0}\right)}+B\right)=0
$$

where

$$
B=\left(\begin{array}{cc}
\left(m_{11}-h\right)(1 / r-1) & m_{12}(1 / r-1) \\
m_{21}(1 / r-1) & \left(m_{22}-h\right)(1 / r-1)
\end{array}\right)
$$


Since $r>1$, by (5.9) and Lemma 4.6 we get

$$
\sigma_{1}\left(\mathscr{L}+M_{\left(u_{0}, \nu_{0}\right)}+B\right)>\sigma_{1}\left(\mathscr{L}+M_{\left(u_{0}, \nu_{0}\right)}\right)>0
$$

contradicting (5.10).

The following result provides us with a sufficient condition for $\sigma_{1}\left(\mathscr{L}+M_{\left(u_{0}, \nu_{0}\right)}\right)>0$ to hold.

PROPOSITION 5.3. Assume that $m=n, a(x), d(x)>0$ for all $x \in \bar{\Omega}, \lambda$ and $\mu$ satisfy (3.2) and that for any $\left(u_{0}, v_{0}\right)$ coexistence state of (1.3),

$$
\left(\frac{b}{a}\right)_{M}\left(\frac{c}{d}\right)_{M}\left(\frac{u_{0}}{v_{0}}\right)_{M}^{(2-m) / m}\left(\frac{v_{0}}{u_{0}}\right)_{M}^{(2-m) / m}<1
$$

Then (1.3) possesses a unique coexistence state.

Proof. Let $\Phi:=\left(\alpha u_{0}^{1 / m},-\beta v_{0}^{1 / m}\right) \in\left(C^{2}(\Omega) \cap C_{0}^{0}(\bar{\Omega})\right)^{2}$, with $\alpha, \beta>0$ to be chosen. We will show that $\Phi$ is a supersolution in the sense of Definition 4.1 of $\mathscr{L}+M_{\left(u_{0}, \nu_{0}\right)}$ if (5.11) holds. Proposition 4.3 and Theorem 5.2 will complete the proof.

First, observe that $\Phi>0$. In order to show that $\Phi$ is a supersolution of $\mathscr{L}+M_{\left(u_{0}, v_{0}\right)}$ we have to prove that (for the first equation)

$$
L_{1}\left(\alpha u_{0}^{1 / m}\right)+m_{11}(x) \alpha u_{0}^{1 / m}+m_{12}(x)\left(-\beta v_{0}^{1 / m}\right)>0
$$

where $m_{11}$ and $m_{12}$ are defined in (5.7). Taking into account the fact that

$$
L_{1}\left(u_{0}^{1 / m}\right)=\frac{1}{m} u_{0}^{1 / m-1}\left[\left(1-\frac{1}{m}\right) u_{0}^{-1} \sum_{i, j=1}^{N} a_{i j}^{1} D_{i} u_{0} D_{j} u_{0}+L_{1} u_{0}\right],
$$

to prove (5.12) it suffices that

$$
a(x) u_{0}^{2 / m-1}>b(x) v_{0}^{2 / m-1} \frac{\beta}{\alpha}, \quad \text { for all } x \in \Omega .
$$

Analogously, for the second equation it is sufficient that

$$
d(x) v_{0}^{2 / m-1}>c(x) u_{0}^{2 / m-1} \frac{\alpha}{\beta}, \quad \text { for all } x \in \Omega
$$

Now, by (5.11) it is easy to show that there exist $\alpha$ and $\beta$ satisfying the above inequalities. 
The following result provides us with another sufficient condition to obtain a uniqueness result.

PROPOSITION 5.4. Assume that $\lambda$ and $\mu$ satisfy (3.2) and that for any coexistence state $\left(u_{0}, v_{0}\right)$ of $(1.3)$ the following inequalities hold for all $x \in \Omega$ :

$$
\begin{gathered}
\lambda\left(1-\frac{1}{m}\right)+a(x) u_{0}^{1 / m}(x)\left(\frac{2}{m}-1\right)>b(x)\left(1+\frac{1}{n}-\frac{1}{m}\right) v_{0}^{1 / n}(x), \\
\mu\left(1-\frac{1}{n}\right)+d(x) v_{0}^{1 / n}(x)\left(\frac{2}{n}-1\right)>c(x)\left(1+\frac{1}{m}-\frac{1}{n}\right) u_{0}^{1 / m}(x) .
\end{gathered}
$$

Then (1.3) possesses a unique coexistence state.

PROOF. Taking $\Phi:=\left(u_{0},-v_{0}\right)$, it suffices to prove that $\Phi \succ 0$ is a supersolution of $\mathscr{L}+M_{\left(u_{0}, v_{0}\right)}$ provided that (5.13) holds and we again apply Proposition 4.3 and Theorem 5.2. For the second equation, $\Phi$ is a supersolution if

$$
L_{2}\left(-v_{0}\right)+m_{21}(x)\left(u_{0}\right)+m_{22}(x)\left(-v_{0}\right)<0,
$$

where $m_{21}$ and $m_{22}$ are defined in (5.7). For observe that

$$
\begin{aligned}
& L_{2}\left(-v_{0}\right)+m_{21}(x)\left(u_{0}\right)+m_{22}(x)\left(-v_{0}\right) \\
& \quad=v_{0}^{1 / n}\left(\mu\left(\frac{1}{n}-1\right)+d(x) v_{0}^{1 / n}\left(1-\frac{2}{n}\right)+c(x) u_{0}^{1 / m}\left(1+\frac{1}{m}-\frac{1}{n}\right)\right)<0,
\end{aligned}
$$

provided that (5.13) holds. Similarly we can reason with the first equation.

We will now use the upper estimates of (3.3) and (3.4) giving sufficient conditions for the uniqueness of a coexistence state in terms of several coefficients involved in the model setting.

COROLlaRY 5.5. Assume that $m=n, a(x), d(x)>0$ for $x \in \bar{\Omega}, \lambda$ and $\mu$ satisfy (3.2) and

$$
\left(\frac{\left(e_{1}\right)_{M}^{1 /(m-1)}}{\varepsilon_{1}} \frac{\left(e_{2}\right)_{M}^{1 /(m-1)}}{\varepsilon_{2}}\right)^{(2-m) / m}\left(\frac{e_{1}}{\varphi_{2}}\right)_{M}^{(2-m) / m}\left(\frac{e_{2}}{\varphi_{1}}\right)_{M}^{(2-m) / m}(\lambda \mu)^{(2-m) /(m-1)}<\frac{a_{L} d_{L}}{b_{M} c_{M}},
$$

where $\varepsilon_{1}$ and $\varepsilon_{2}$ are defined in (3.5). Then (1.3) possesses a unique coexistence state.

Proof. By (3.3) and (3.4) we have that

$$
\left(\frac{u_{0}}{v_{0}}\right)_{M} \leq \frac{\lambda^{m /(m-1)}\left(e_{1}\right)_{M}^{1 /(m-1)}}{\varepsilon_{2}}\left(\frac{e_{1}}{\varphi_{2}}\right)_{M}
$$


and

$$
\left(\frac{v_{0}}{u_{0}}\right)_{M} \leq \frac{\mu^{m /(m-1)}\left(e_{2}\right)_{M}^{1 /(m-1)}}{\varepsilon_{1}}\left(\frac{e_{2}}{\varphi_{1}}\right)_{M},
$$

and so (5.11) is satisfied if (5.14) holds. It suffices to apply Proposition 5.3.

COROLLARY 5.6. Assume that some of the following sets of inequalities, (1) to (4), hold:

(1) If $1<m, n \leq 2$,

$$
\begin{aligned}
& b_{M}\left(1+\frac{1}{n}-\frac{1}{m}\right) \mu^{1 /(n-1)}\left(e_{2}\right)_{M}^{1 /(n-1)}<\lambda\left(1-\frac{1}{m}\right), \\
& c_{M}\left(1+\frac{1}{m}-\frac{1}{n}\right) \lambda^{1 /(m-1)}\left(e_{1}\right)_{M}^{1 /(m-1)}<\mu\left(1-\frac{1}{n}\right) .
\end{aligned}
$$

(2) If $1<n \leq 2$ and $m>2$,

$$
\begin{aligned}
& b_{M}\left(1+\frac{1}{n}-\frac{1}{m}\right) \mu^{1 /(n-1)}\left(e_{2}\right)_{M}^{1 /(n-1)}+\left(1-\frac{2}{m}\right) a_{M} \lambda^{1 /(m-1)}\left(e_{1}\right)_{M}^{1 /(m-1)}<\lambda\left(1-\frac{1}{m}\right), \\
& c_{M}\left(1+\frac{1}{m}-\frac{1}{n}\right) \lambda^{1 /(m-1)}\left(e_{1}\right)_{M}^{1 /(m-1)}<\mu\left(1-\frac{1}{n}\right) .
\end{aligned}
$$

(3) If $1<m \leq 2$ and $n>2$,

$$
\begin{aligned}
& c_{M}\left(1+\frac{1}{m}-\frac{1}{n}\right) \lambda^{1 /(m-1)}\left(e_{1}\right)_{M}^{1 /(m-1)}+\left(1-\frac{2}{n}\right) d_{M} \mu^{1 /(n-1)}\left(e_{2}\right)_{M}^{1 /(n-1)}<\mu\left(1-\frac{1}{n}\right), \\
& b_{M}\left(1+\frac{1}{n}-\frac{1}{m}\right) \mu^{1 /(n-1)}\left(e_{2}\right)_{M}^{1 /(n-1)}<\lambda\left(1-\frac{1}{m}\right) .
\end{aligned}
$$

(4) If $m>2$ and $n>2$,

$$
\begin{aligned}
& b_{M}\left(1+\frac{1}{n}-\frac{1}{m}\right) \mu^{1 /(n-1)}\left(e_{2}\right)_{M}^{1 /(n-1)}+\left(1-\frac{2}{m}\right) a_{M} \lambda^{1 /(m-1)}\left(e_{1}\right)_{M}^{1 /(m-1)}<\lambda\left(1-\frac{1}{m}\right), \\
& c_{M}\left(1+\frac{1}{m}-\frac{1}{n}\right) \lambda^{1 /(m-1)}\left(e_{1}\right)_{M}^{1 /(m-1)}+\left(1-\frac{2}{n}\right) d_{M} \mu^{1 /(n-1)}\left(e_{2}\right)_{M}^{1 /(n-1)}<\mu\left(1-\frac{1}{n}\right) .
\end{aligned}
$$

Then (1.3) possesses a unique coexistence state.

Proof. Reasoning as in the proof of Corollary 5.5, it is sufficient to apply (3.3), (3.4) and Proposition 5.4.

REMARK 6. (1) Observe that when $m=1,(5.11)$ is the condition obtained in [27, Theorem 4.2] and [21, Theorem 4.8]. Moreover, when $m=n$, and $a$ and $d$ are positive, we obtain uniqueness provided that $b_{M}$ or $c_{M}$ is small. 
(2) The $(\lambda, \mu)$-regions defined in Corollary 5.6 are subsets of the coexistence region obtained in Theorem 3.2. Similar conditions to those imposed in Figure 1 assure the existence of these subregions.

\section{Conclusions}

We have studied the set of nonnegative solutions of a spatially heterogeneous LotkaVolterra competition model with degenerate diffusion. Basically, we have found three differences with respect to the non-degenerate (linear) case:

(1) In the degenerate case all the nonnegative solutions are bounded, unlike the linear case in which a priori bounds are lost for some values of the data of the problem.

(2) In the degenerate case a new kind of nonnegative solution appears: nonnegative and nontrivial solutions that vanish in a region of the habitat of the species. We obtain sufficient conditions in terms of some parameters involved in the setting of the model ensuring the existence or non-existence of such solutions.

(3) Unlike the non-degenerate case, in our model when the competition between the species is "strong" neither species drives the other to extinction.

Finally, we have obtained the uniqueness of the positive solution of the problem under some conditions on the data of the problem.

\section{Acknowledgements}

The author is grateful to Professor M. Delgado for his helpful comments. He thanks MCYT of Spain for research support under grants BFM2000-0797 and BFM200306446. Finally, he would like to acknowledge the anonymous referee for useful remarks which improved this paper.

\section{References}

[1] H. Amann, "On the existence of positive solutions of nonlinear elliptic boundary value problems", Indiana Univ. Math. J. 21 (1971) 125-146.

[2] H. Amann, "Fixed point equations and nonlinear eigenvalue problems in ordered Banach spaces", SIAM Rev. 18 (1976) 620-709.

[3] C. Bandle, M. A. Pozio and A. Tesei, "The asymptotic behaviour of the solutions of degenerate parabolic equations", Trans. Amer. Math. Soc. 303 (1987) 487-501.

[4] H. Berestycki and P. L. Lions, "Some applications of the method of sub and supersolutions", in Bifurcation and nonlinear eigenvalue problem, Springer Lectures Notes 782, (Springer, 1980) 16-41. 
[5] M. Bertsch and R. Rostamian, "The principle of linearized stability for a class of degenerate diffusion equations", J. Differential Equations 57 (1985) 373-405.

[6] J. Blat and K. J. Brown, "Bifurcation of steady-state solutions in predator-prey and competition systems", Proc. Roy. Soc. Edinburgh Sect. A 97 (1984) 21-34.

[7] R. S. Cantrell and C. Cosner, "On the steady-state problem for the Volterra-Lotka competition model with diffusion", Houston J. Math. 13 (1987) 337-352.

[8] R. S. Cantrell and C. Cosner, "Should a park be an island?", SIAM J. Math. Anal. 53 (1993) 219-252.

[9] C. Cosner and A. C. Lazer, "Stable coexistence states in the Volterra-Lotka competition model with diffusion", SIAM J. Appl. Math. 44 (1984) 1112-1132.

[10] E. N. Dancer, "On the indices of fixed points of mappings in cones and applications", J. Math. Anal. Appl. 91 (1983) 131-151.

[11] E. N. Dancer, "On positive solutions of some pairs of differential equations, part I", Trans. Amer. Math. Soc. 284 (1984) 729-743.

[12] E. N. Dancer, "On the existence and uniqueness of positive solutions for competing species models with diffusion", Trans. Amer. Math. Soc. 326 (1991) 828-859.

[13] M. Delgado and A. Suárez, "On the existence of dead cores for degenerate Lotka-Volterra models", Proc. Roy. Soc. Edinburgh Sect. A 130 (2000) 743-766.

[14] M. Delgado and A. Suárez, "On the structure of the positive solutions of the logistic equation with nonlinear diffusion", J. Math. Anal. Appl. 268 (2002) 200-216.

[15] M. Delgado and A. Suárez, "Stability and uniqueness for cooperative degenerate Lotka-Volterra model", Nonlinear Anal. 49 (2002) 757-778.

[16] J. I. Díaz and J. Hernández, "On the existence of a free boundary for a class of reaction-diffusion systems", SIAM. J. Math. Anal. 15 (1984) 670-685.

[17] Y. Du, "Effects of a degeneracy in the competition model: parts I and II", J. Differential Equations 181 (2002) 92-132 and 133-164.

[18] Y. Du and K. J. Brown, "Bifurcation and monotonicity in competition reaction-diffusion systems", Nonlinear Anal. 23 (1994) 1-13.

[19] J. C. Eilbeck, J. E. Furter and J. López-Gómez, "Coexistence in the competition model with diffusion", J. Differential Equations 107 (1994) 96-139.

[20] J. E. Furter and J. López-Gómez, "On the existence and uniqueness of coexistence states for the Lotka-Volterra competition model with diffusion and spatially dependent coefficients", Nonlinear Anal. 25 (1995) 363-398.

[21] J. E. Furter and J. López-Gómez, "Diffusion-mediated permanence problem for an heterogeneous Lotka-Volterra competition model", Proc. Roy. Soc. Edinburgh Sect. A 127 (1997) 281-336.

[22] M. E. Gurtin and R. C. MacCamy, "On the diffusion of biological populations", Math. Biosci. 33 (1977) 35-49.

[23] A. W. Leung and G. Fan, "Existence of positive solutions for elliptic systems--degenerate and nondegenerate ecological models", J. Math. Anal. Appl. 151 (1990) 512-531.

[24] L. Li and R. Logan, "Positive solutions to general elliptic competition models", Differential Integral Equations 4 (1991) 817-834.

[25] J. López-Gómez, "On the structure of the permanence region for the competing species models with general diffusivities and transports effects", Discrete Contin. Dynam. Systems 2 (1996) 525-542.

[26] J. López-Gómez, "Coexistence and metacoexistence states in competing species model", Houston J. Math. 29 (2003) 483-536.

[27] J. López-Gómez and J. C. Sabina de Lis, "Coexistence states and global attractivity for some convective diffusive competing species models", Trans. Amer. Math. Soc. 347 (1995) 3797-3833.

[28] A. Cañada and J. L. Gámez, "Elliptic systems with nonlinear diffusion in population dynamics", Differential Equations Dynam. Systems 3 (1995) 189-204. 
[29] T. Namba, "Density-dependent dispersal and spatial distribution of a population", J. Theor. Biol. 86 (1980) 351-363.

[30] M. A. Pozio and A. Tesei, "Degenerate parabolic problems in population dynamics", Japan J. Appl. Math. 2 (1985) 351-380.

[31] M. A. Pozio and A. Tesei, "Support properties of solutions for a class of degenerate parabolic problems", Comm. Partial Differential Equations 12 (1987) 47-75.

[32] W. H. Ruan and C. V. Pao, "Positive steady-state solutions of a competing reaction-diffusion system", J. Differential Equations 117 (1995) 401-427. 